\title{
A CMOS Current Driver With Built-in Common- Mode Signal Reduction Capability for EIT
}

\author{
$\mathrm{Yu} \mathrm{Wu}^{1}$, Dai Jiang ${ }^{1}$, Peter Langlois ${ }^{1}$, Richard Bayford ${ }^{2}$, and Andreas Demosthenous ${ }^{1}$ \\ ${ }^{1}$ Department of Electronic and Electrical Engineering, University College London, Torrington Place, London WC1E 7JE, UK \\ ${ }^{2}$ Department of Natural Sciences, Middlesex University, The Burroughs, London, NW4 4BT, UK \\ e-mail: yu.wu.09@ucl.ac.uk; a.demosthenous@ucl.ac.uk
}

\begin{abstract}
This paper presents an integrated fully differential current driver for wearable multi-frequency electrical impedance tomography (EIT). The integrated circuit (IC) comprises a wideband current driver (up to $500 \mathrm{kHz}$ ) functioning as the master for current sourcing, and a differential voltage receiver with common-mode feedback configuration as the slave for current sinking. The IC is fabricated in a $0.18-\mu \mathrm{m}$ CMOS technology. It operates from $\pm 1.65 \mathrm{~V}$ power supplies and occupies a total die area of less than $0.05 \mathrm{~mm}^{2}$. The current driver has a measured output impedance of $750 \mathrm{k} \Omega$ at $500 \mathrm{kHz}$ and provides a common-mode signal reduction of $32 \mathrm{~dB}$ at $500 \mathrm{kHz}$. The application of the IC in a wearable EIT lung monitoring system is presented.
\end{abstract}

\section{INTRODUCTION}

Electrical impedance tomography (EIT) is a non-invasive, radiation-free imaging technique that has been widely used in many clinical studies such as brain activity detection, hand gesture recognition, breast cancer detection and lung aeration monitoring. Recent studies [1], [2] show that EIT can facilitate real time dynamic monitoring of lung aeration and improve the breathing control of both children and preterm babies by indicating areas of lung that are subject to over inflation or lung collapse. Currently, especially for preterm babies, there is no monitoring device available to detect and give early warning of respiratory failure.

EIT images an object by injecting ac currents to the subject under test (SUT) via surface electrodes, while measuring the differential voltages across other adjacent pairs of electrodes. As the current mostly concentrates around its injected source, the potential developed becomes smaller as the measuring electrodes become further away from the current source. To drive the load differentially the current driver needs to be floating. Any mismatch between the source and sink side of the driving circuits generates a common-mode signal that is superimposed on the differential signal at the same frequency. To obtain a high measurement accuracy $(0.1 \%$ error $),>100$ $\mathrm{dB}$ CMRR for the front-end instrumentation amplifier is suggested [3]; however using complex switching networks in front of the instrumentation amplifier for electrodes multiplexing, such a high CMRR is often difficult to achieve.

The most commonly adapted current driver in EIT systems is the Howland circuit. The matching between source and sink on this circuit relies entirely on the matching of its feedback

This work was funded by the European Commission under grant agreement no. 668259 - CRADL (www.cradlproject.org). resistors. Current drivers based on push-pull current mirrors controlled by a voltage input are reported in [4]-[6] for biomedical application ICs. Several custom current driver ICs using feedback have also been reported [7], [8], offering better performance than open-loop systems. However, the source and sink sides of those drivers are relatively independent, and the matching is heavily dependent on the feedback resistor $R_{S}$ and the input voltage. In [9] and [10] frequency selective common-mode feedback (CMFB) is implemented to minimize common-mode errors which degrade the performance of practical EIT systems. However, the use of frequency selective CMFB greatly increases the circuit complexity.

An alternative and much simpler arrangement for CMFB operating in conjunction with a wideband current driver is described in this paper. The current driver acts as the master for current sourcing. It provides an output current of up to $1 \mathrm{~mA}_{\mathrm{p}-\mathrm{p}}$ and has an output impedance of in excess $750 \mathrm{k} \Omega$ at $500 \mathrm{kHz}$. A slave differential voltage receiver (DVR) sinks the exact amount of current sourced by the master through CMFB. Using feedback, the DVR inherits the high output impedance characteristic of the master with low circuit complexity.

The rest of the paper is organized as follows. Section II presents the IC system architecture and Section III describes the circuit details of its building blocks. Section IV shows the measured IC performance. Conclusions are drawn in Section V.

\section{SYSTEM ARCHITECTURE}

To visualize the issue of common-mode error and aid the analysis, a homogenous circle SUT has been simplified to the equivalent circuit shown on Fig. 1. With zero mismatches, any two voltages developed at two mirrored locations on the SUT with respect to the injecting electrode pair are expected to be fully differential. However, given that, for example the human thorax is neither symmetrical nor homogenous, the commonmode signal needs to be measured from the injecting electrode pairs. Thus the SUT is equivalent to a load with impedance value of $Z_{L}$ seen by the current driver. The electrode is modelled as $Z_{e}$, and at an injecting of frequency of a few hundreds of $\mathrm{kHz}$ the value of $Z_{e}$ becomes negligible.

In Fig. 1 (a), when mismatches occur as shown in red, a mismatched current $\Delta I$ is generated and flows through the high impedance node $Z_{o}$ of the current driver. This results in two possible scenarios: 1) The output of the current driver is saturated, especially for large mismatch errors; 2) With small 
mismatch errors, low $Z_{o}$ or benefiting from a high voltage compliance, the current driver may not be saturated, but a large common-mode signal of $\Delta I \times \frac{z_{O}}{2}$ is generated and added to the small differential signals which then has to be accommodated by CMRR of the instrumentation amplifier.
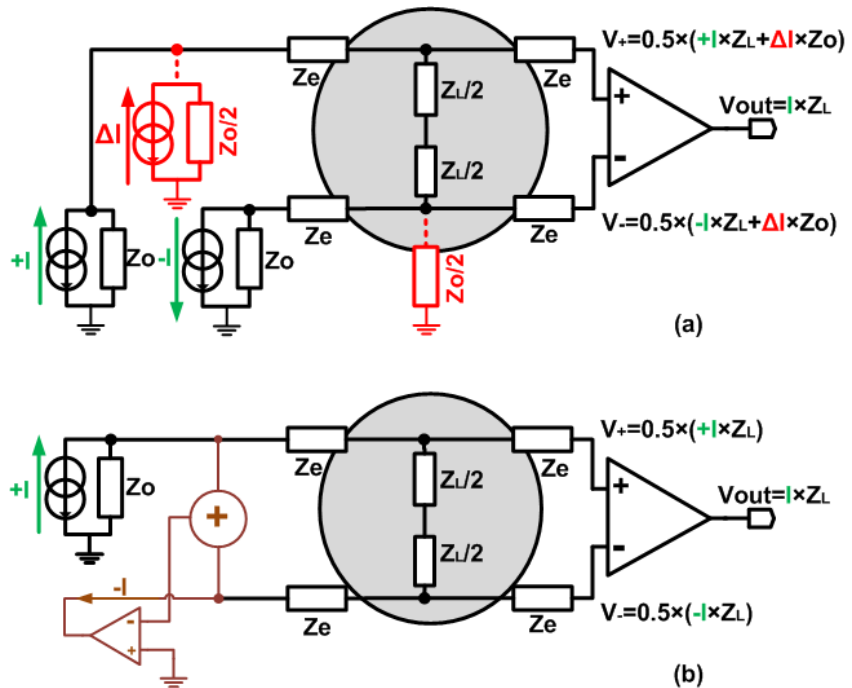

Fig. 1. (a) Current driver with a $\Delta I$ mismatched current flowing through the load $Z_{o}$; (b) Current driver with common-mode feedback to reduce the effect of mismatches.

Fig.1 (b) shows the proposed CMFB method, which provides an active feedback current sink to solve this issue. Depending on the application, the feedback may be applied with both the source and sink side of the differential current driver present and only compensate for the mismatch current $\Delta I$. Alternatively, it can completely replace the original sink side, providing enough drivability for differential output currents.

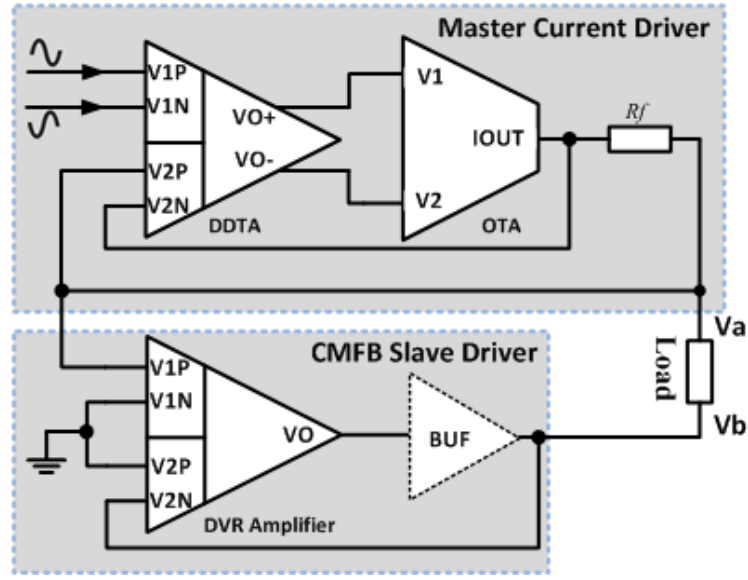

Fig. 2. Circuit architecture of current driver with CMFB.

The circuit architecture is shown in Fig. 2; the master current driver consists of a differential difference transconductance amplifier (DDTA) followed by an operational transconductance amplifier (OTA) to enhance the overall transconductance. The DDTA measures the output current through the sensor resistor, $R_{f}=250 \Omega$, and forms a linear current feedback. The DVR measures the voltage across the load and, through feedback, forces the common-mode voltage across the load to be zero. Consequently, it sinks the current sourced by the master driver to form a complete current path. As the feedback is continuous in time, the DVR is able to feedback multi-frequency injection. A voltage buffer is optional for driving low impedance loads.

\section{CIRCUIT IMPLEMENTATION}

\section{A. Master Current Driver}

The master current driver on the top half of Fig. 2 has a transfer function defined by

$$
G m_{\text {driver }}=\frac{\text { Iout }}{\mathrm{V} 1 \mathrm{P}-\mathrm{V} 1 \mathrm{~N}}=\frac{G m_{\text {loop }}}{1+G m_{\text {loop }} \times R_{f}}
$$

and

$$
G m_{\text {loop }}=A_{\text {ol-ddta }} \times G m_{\text {ota }}
$$

where the $A_{o l-d d t a}$ is the open loop gain of the DDTA, and $G m_{\text {ota }}$ is the trans-conductance of the OTA stage. To ensure an accurate feedback where the $G m_{\text {driver }} \geq 3.99 \mathrm{~mA} / \mathrm{V}$, the minimum $G m_{\text {loop }}$ required is $1.6 \mathrm{~A} / \mathrm{V}$.

The transistor level schematic of the DDTA is shown in Fig. 3 and the open loop gain is defined as

$$
A_{o l-d d t a} \approx \beta \times g m_{\text {in }} \times\left\{\frac{1}{\frac{1}{\left(g m_{6} \times \frac{1}{g_{06}} \times \frac{1}{g_{07}}\right)}+\frac{1}{\left(g m_{8} \times \frac{1}{g_{08}} \times \frac{1}{g_{09}}\right)}}\right\}
$$

where $g_{o}$ is the admittance at the drain of each labelled transistor, $\beta$ is the transistor $W / L$ ratio between $M 5$ and $M 6$, and $g m_{\text {in }}$ is the transconductance of $M 1$ in combination with the source-degeneration transistors $M a, M b, M c$ and $M d$ to improve the input linear range.

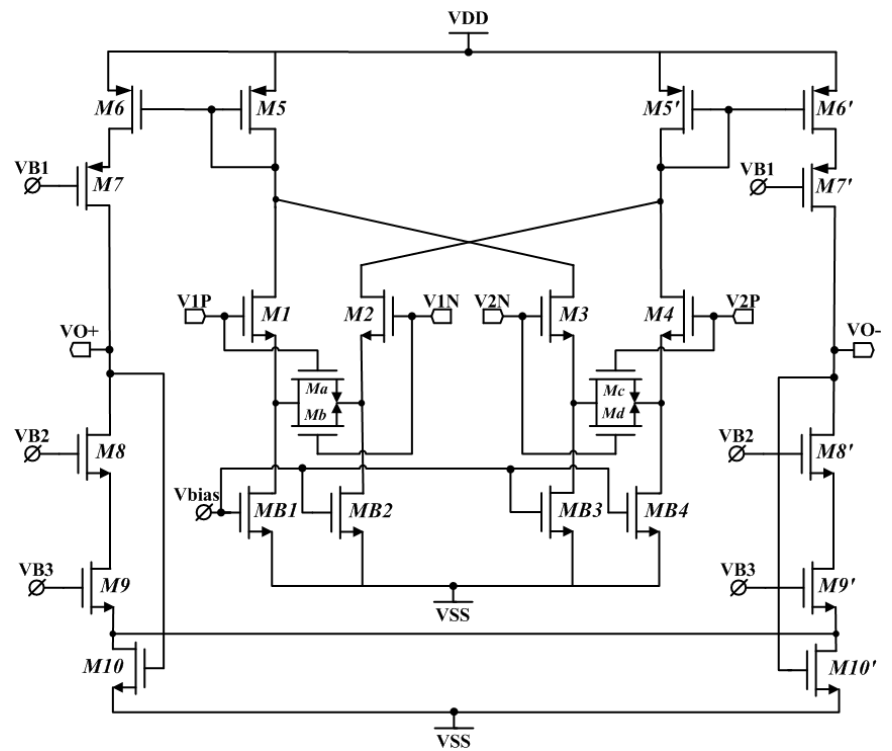

Fig. 3. DDTA circuit schematic.

Transistors $M 10$ and $M 10^{\prime}$ provide CMFB to stabilize the dc levels at the output stage. Load capacitors can be added at the output $\mathrm{VO}+$ and $\mathrm{VO}-$ to set the dominant pole of DDTA to improve the phase margin of the current driver. 
A classic symmetrical OTA has been designed. This circuit operates in combination with the DDTA to provide the overall $G m_{\text {loop }}$, and the $G m_{\text {ota }}$ can be set by the transconductance of the input pair and the $\beta$ ratio factor. With negative current feedback, the output impedance of the current driver is the impedance $r o_{o t a}$ seen at IOUT of the OTA multiplied by an amplification factor of $G m_{\text {loop }} \times R_{f}$.

\section{B. Slave Driver: CMFB Current Sink}

The schematic of the DVR amplifier is shown in Fig. 4. It has a similar input stage to the DDTA, but with a diodeconnected M5 transistor to convert the output to a single node. Two-stage amplification is used with Miller compensation and nulling resistor to improve gain and stability. The transfer function of the circuit is

$$
\mathrm{VO} \approx A_{\text {loop }} \times\{(V 1 P-V 1 N)-(V 2 P-V 2 N)\}
$$

where $A_{\text {loop }}=\frac{g m_{1}}{g_{01}+g_{02}} \times \frac{g m_{7}}{g_{07}+g_{08}}$, and by connecting the circuit as shown in Fig. 2, the closed loop function can be written as

$$
V_{b}=V_{a} \times\left(\frac{1-A_{\text {loop }}}{A_{\text {loop }}}\right) \approx-V_{a} .
$$

From (6), the circuit can also be seen as a resistor-less inverting unity gain amplifier, and by centre-tapping the load to a virtual ground, the current in the load can be written as

$$
I_{\text {source }}=\frac{\mathrm{Va}}{2 \times R_{l}}=\frac{-\mathrm{Va}}{2 \times R_{l}}=-I_{\text {sink }} \text {. }
$$

The gain-bandwidth of the DVR should be higher than $28 \mathrm{MHz}$ to give a minimum $1^{\circ}$ phase delay at $500 \mathrm{kHz}$.

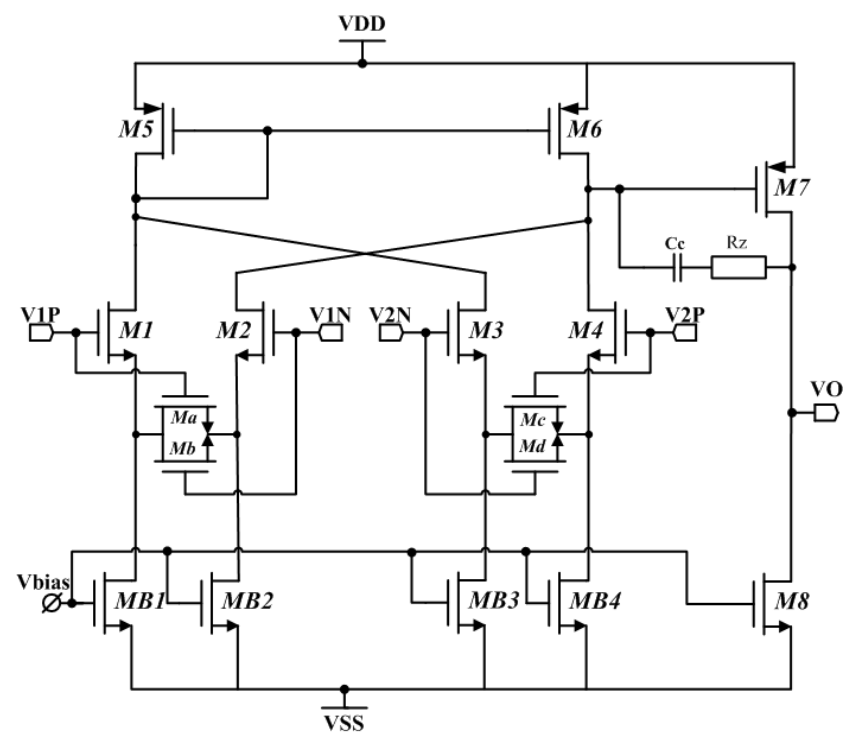

Fig. 4. Differential voltage receiver circuit schematic.

\section{Measured Results}

The IC was fabricated in a $0.18-\mu \mathrm{m}$ CMOS process technology. Fig. 5 shows the microphotograph of the chip; it occupies an area of $200 \mu \mathrm{m} \times 250 \mu \mathrm{m}$. The effect of CMFB has been tested by measuring the common-mode voltage across a $2 \mathrm{k} \Omega$ load at $500 \mathrm{kHz}$, firstly using two individual master current drivers (using two ICs) each working in source and sink configuration driven by a precision differential sinusoidal voltage input and employing $0.1 \%$ tolerance off-chip feedback resistors then using the complete CMFB current driver IC to compare the results. As shown in Fig. 6, the common-mode signal is reduced by $32 \mathrm{~dB}(97.5 \%$ reduction). Fig. 7 shows the CMFB current driver injecting a multi-tone signal with a THD of $43.7 \mathrm{~dB}$; the source and sink are fully differential with only $1^{\circ}$ phase error.

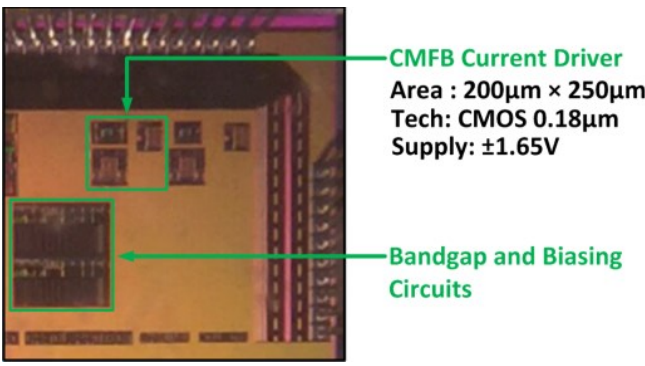

Fig. 5. Die microphotograph of the IC.

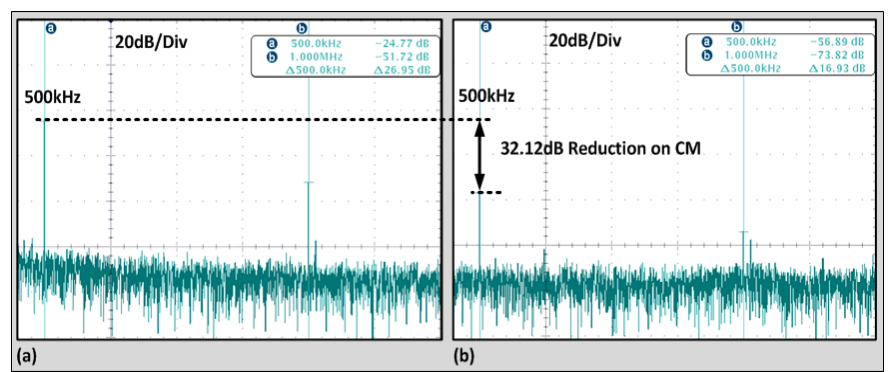

Fig. 6. (a) Common-mode signal measured with two independent current driver ICs. The output is shunt with a $10-\mathrm{k} \Omega$ resistor to prevent saturation; (b) Common-mode signal measured with the CMFB current driver IC.
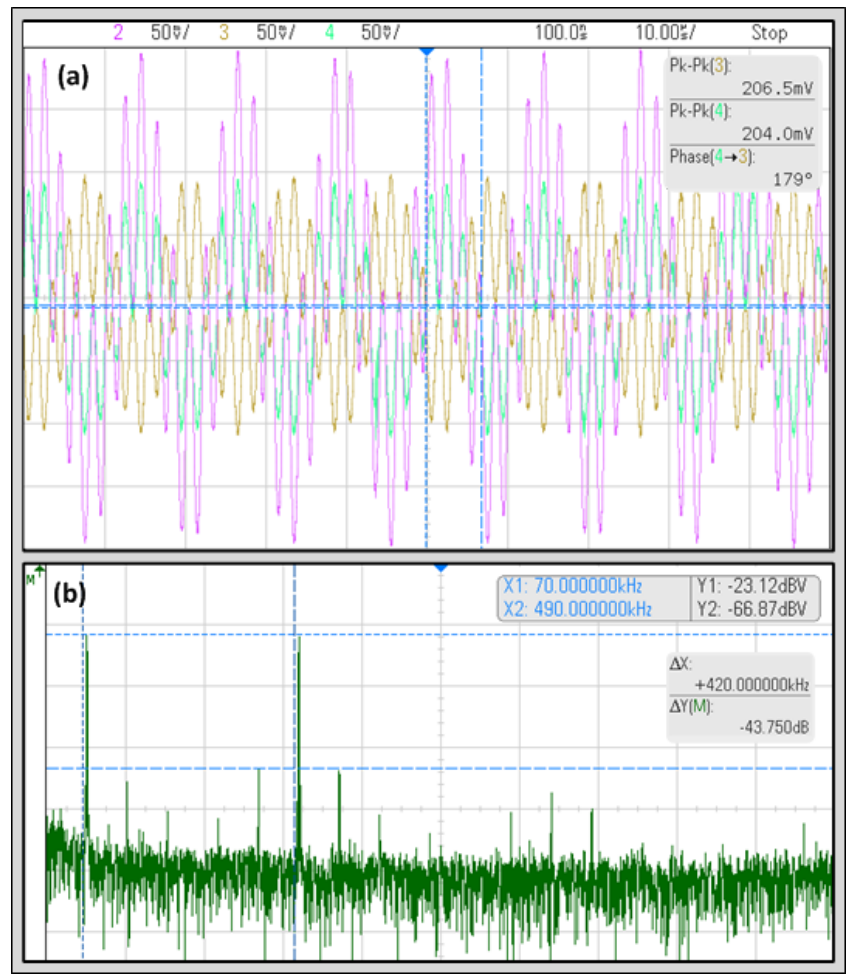

Fig. 7. Multi-frequency injection. Channel 3 and 4 are the voltages measured at each side of the load and channel 2 is the differential voltage across the load. 


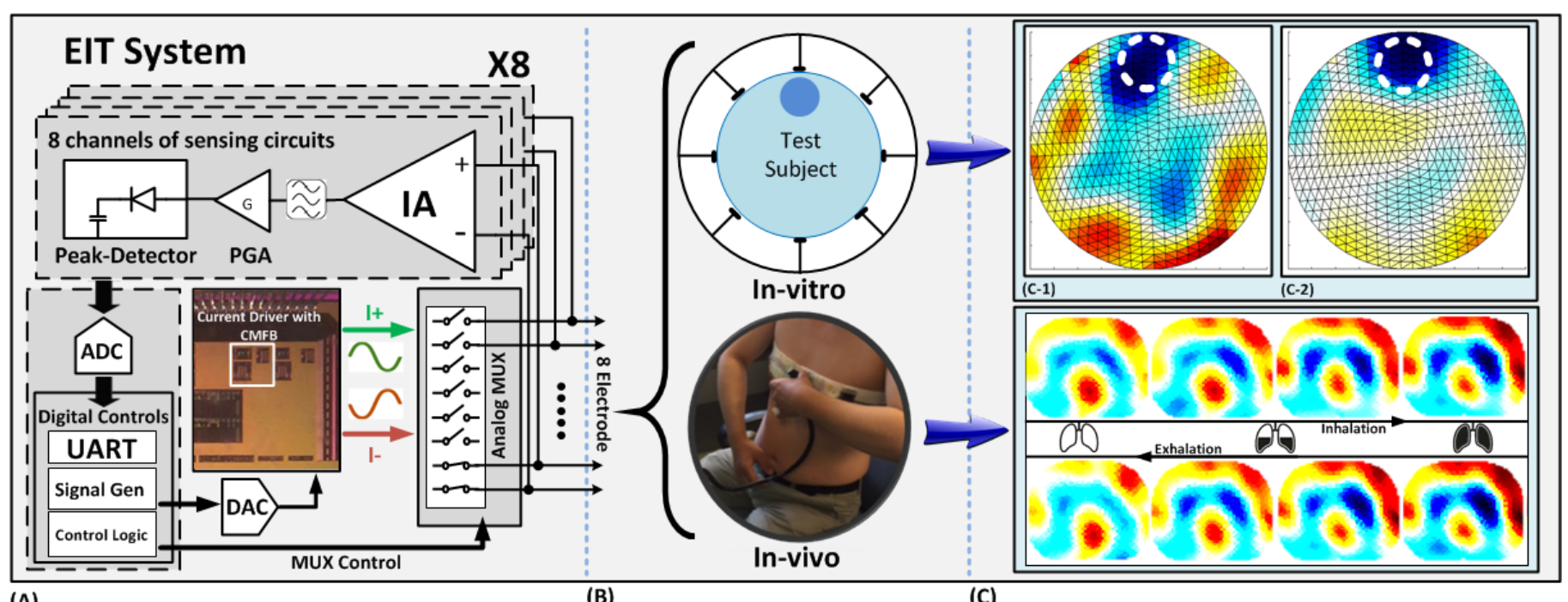

Fig. 8. (A) An 8 electrode wearable EIT system using the chip as the current driver. (B) In-vitro and in-vivo test setup. (C) C-1: In-vitro test results using two master current drivers. C-2: In-vitro test results using the CMFB current driver IC and in-vivo test results from imaging the lung function of a human volunteer.

Fig. 8 shows an 8 electrode EIT system using the IC as the current driver. In-vitro testing used a circular electrode tank filled up with saline solution into which an object was placed to image. Fig. 8(c) shows that the image in $\mathrm{C}-2$ produced with the CMFB method is clearer (the object is more distinguishable and free of other artefacts) compared to the image in C-1 which did not use CMFB. In-vivo testing allowed the respiration cycle of the lung of a volunteer to be captured in real-time at 20 frame/s. The dark blue area in Fig. 8(c) (see lower images) indicates the air inflated lung region, and as the volunteer inhales or exhales, the area of those regions increases or decreases accordingly.

Table I summarises the performance of the CMFB current driver IC and provides a comparison with the state of the art.

TABLE I

COMPARISON WITH OTHER CURRENT DRIVERS

\begin{tabular}{|c|c|c|c|c|c|c|c|}
\hline & This work & [4] & [5] & [6] & [7] & [9] & [10] \\
\hline Bandwidth & $500 \mathrm{kHz}$ & $20 \mathrm{kHz}$ & $256 \mathrm{kHz}$ & $100 \mathrm{kHz}$ & $500 \mathrm{kHz}$ & $250 \mathrm{kHz}$ & $800 \mathrm{kHz}$ \\
\hline $\begin{array}{c}\text { Output } \\
\text { Impedance }\end{array}$ & $\begin{array}{c}750 \mathrm{k} \Omega \\
@ 500 \mathrm{kHz}\end{array}$ & - & - & $\begin{array}{c}100 \mathrm{k} \Omega \\
@ 100 \mathrm{kHz}\end{array}$ & $\begin{array}{c}372 \mathrm{k} \Omega \\
@ 500 \mathrm{kHz}\end{array}$ & $z$ & $\begin{array}{l}50 \mathrm{k} \Omega \\
@ \mathrm{DC}\end{array}$ \\
\hline $\begin{array}{c}\text { Max Current } \\
\& \text { THD }\end{array}$ & $\begin{array}{l}1 \mathrm{mApp} \\
42 \mathrm{~dB}\end{array}$ & $\begin{array}{l}117 \mu \mathrm{App}, \\
\text { pseudo-sine }\end{array}$ & $\begin{array}{l}254 \mu \mathrm{App}, \\
\text { pseudo-sine }\end{array}$ & $\begin{array}{c}400 \mu \mathrm{App}, \\
54 \mathrm{~dB}\end{array}$ & $\begin{array}{c}5 \mathrm{mApp} \\
46 \mathrm{~dB}\end{array}$ & - & - \\
\hline $\begin{array}{l}\text { Common } \\
\text { Mode } \\
\text { Reduction }\end{array}$ & $\begin{array}{c}97.5 \% \\
\text { (voltage } \\
\text { feedback) }\end{array}$ & No & No & No & No & $65 \%$ & $\begin{array}{c}97.2 \% \\
\text { (voltage } \\
\text { feedback) }\end{array}$ \\
\hline $\begin{array}{l}\text { Supply } \\
\text { Voltage }\end{array}$ & $3.3 \mathrm{~V}$ & $1.2 \mathrm{~V}$ & $3.3 \mathrm{~V}$ & $1.8 \mathrm{~V}$ & $18 \mathrm{~V}$ & $5 \mathrm{~V}$ & - \\
\hline Technology & $\begin{array}{l}\text { CMOS } \\
0.18 \mu \mathrm{m}\end{array}$ & $\begin{array}{l}\text { CMOS } \\
0.18 \mu \mathrm{m}\end{array}$ & $\begin{array}{l}\text { CMOS } \\
0.35 \mu \mathrm{m}\end{array}$ & $\begin{array}{l}\text { CMOS } \\
0.18 \mu \mathrm{m}\end{array}$ & $\begin{array}{l}\text { CMOS } \\
0.6 \mu \mathrm{m}\end{array}$ & $\begin{array}{l}\text { CMOS } \\
0.35 \mu \mathrm{m}\end{array}$ & $\begin{array}{l}\text { Discrete } \\
\text { PCB }\end{array}$ \\
\hline
\end{tabular}

\section{CONCLUSION}

A fully integrated, high performance current driver has been implemented in CMOS $0.18 \mu \mathrm{m}$. The inclusion of a continuous CMFB technique has provided a reduction of $97.5 \%$ on the measured common-mode signal. Multifrequency injection has been also demonstrated without the need for any additional frequency selective feedback blocks (as opposed to the designs in [9] and [10]). The CMFB current driver IC has been incorporated in a wearable EIT system. The merit of the CMFB technique has been demonstrated in terms of the quality of the EIT images produced during in-vitro testing. The respiration of the lung has also been successfully imaged during the in-vivo experiment.

\section{REFERENCES}

[1] S. Lehmann, et al, "Global and regional lung function in cystic fibrosis measured by electrical impedance tomography," Pediatric Pulmonology., vol. 51, no. 11, pp. 1191-1199, 2016.

[2] J. L. Hough, A. D. Shearman, H. Liley, C. A. Grant, and A. Schibler, "Lung recruitment and endotracheal suction in ventilated preterm infants measured with electrical impedance tomography," J. Paediatr. Child. Health, vol. 50, no. 11, pp. 884-889, 2014.

[3] J. Rosell, and P. Riu, "Common-mode feedback in electrical impedance tomography, "Clinical Physics and Physiological Measurement, 1992.

[4] N. Van Helleputte et al., "A $345 \mu \mathrm{W}$ multi-sensor biomedical SoC with bio-impedance, 3-channel ECG, motion artifact reduction, and integrated DSP, " IEEE Journal Solid-State Circuits, vol. 50, no. 1, pp. 230-244, Jan. 2015.

[5] M. Guermandi, R. Cardu, E. Franchi Scarselli and R. Guerrieri, "Active electrode IC for EEG and electrical impedance tomography with continuous monitoring of contact impedance," IEEE Transactions Biomedical Circuits and Systems, vol. 9, no. 1, pp. 21-33, Feb. 2015.

[6] S. Hong et al., "A $4.9 \mathrm{~m} \Omega$-sensitivity mobile electrical impedance tomography IC for early breast-cancer detection system," IEEE Journal of Solid-State Circuits, vol. 50, no. 1, pp. 245-257, Jan. 2015.

[7] L. Constantinou, I. F. Triantis, R. Bayford and A. Demosthenous, "High-power CMOS current driver with accurate transconductance for electrical impedance tomography," IEEE Transactions Biomedical Circuits and Systems, vol. 8, no. 4, pp. 575-583, Aug. 2014.

[8] Y. Wu, P. Langlois, R. Bayford and A. Demosthenous, "Design of a CMOS active electrode IC for wearable electrical impedance tomography systems," 2016 IEEE International Symposium on Circuits and Systems (ISCAS), Montreal, QC, 2016, pp. 846-849.

[9] M. Rahal, A. Demosthenous and R. Bayford, "An integrated commonmode feedback topology for multi-frequency bioimpedance imaging," 2009 Proceedings of ESSCIRC, Athens, 2009, pp. 416-419.

[10] P. Langlois, Y. Wu, R. Bayford, and A. Demosthenous, "On the application of frequency selective common-mode feedback for multifrequency EIT," Physiological Measurement, vol. 36, no. 6, 2015. 